\title{
MAJORIZATION-SUBORDINATION THEOREMS FOR LOCALLY UNIVALENT FUNCTIONS. III
}

\author{
BY \\ DOUGLAS MICHAEL CAMPBELL
}

\begin{abstract}
A quantitative majorization-subordination result of Goluzin and Tao Shah for univalent functions is generalized to $\mathfrak{n}_{\infty}$, the linear invariant family of locally univalent functions of finite order $\alpha$. If $f(z)$ is subordinate to $F(z)$ in the open unit disc, $f^{\prime}(0) \geq 0$, and $F(z)$ is in $\mathfrak{u}_{a,} 1.65 \leq \alpha<\infty$, then $f^{\prime}(z)$ is majorized by $F^{\prime}(z)$ in $|z| \leq(\alpha+1)-\left(\alpha^{2}+2 \alpha\right)^{1 / 2}$. The result is sharp.
\end{abstract}

I. Introduction. Let $\subseteq$ denote the set of all normalized analytic univalent functions in the open unit disc $D$. Let $f(z), F(z)$ and $\varphi(z)$ be analytic in $|z|<r$. We say that $f(z)$ is majorized by $F(z)$ in $|z|<r$, if $|f(z)| \leq|F(z)|$ in $|z|<r$. We say that $f(z)$ is subordinate to $F(z)$ in $|z|<r$ if $f(z)=F(\varphi(z))$ where $|\varphi(z)| \leq|z|$ in $|z|<r$.

Let $\mathfrak{u}_{\alpha}$ be the set of all locally univalent $\left(f^{\prime}(z) \neq 0\right)$ analytic functions in $D$ with order $\leq \alpha$ which are of the form $f(z)=z+\cdots$. The family $\mathfrak{u}_{\alpha}$ is known as the universal linear invariant family of order $\alpha$ [4]. A concise summary and introduction to properties of linear invariant families which relate to the following material is contained in [1]. The present paper concludes the proof of results announced in [1].

Majorization-subordination theory begins with Biernacki who showed in 1936 that if $f(z)$ is subordinate in $D$ to $F(z)(F(z) \in S)$, then $f(z)$ is majorized by $F(z)$ in $|z|<1 / 4$. In the succeeding years Goluzin, Tao Shah, Lewandowski and MacGregor examined various related problems but always under the stipulation that the dominant function $F(z)$ is in $\subseteq$ (for greater detail see [1]).

In 1951 Goluzin showed that if $f(z)$ is majorized by a univalent function $F(z)$, then $f^{\prime}(z)$ would be majorized by $F^{\prime}(z)$ in $|z|<0.12$. He conjectured that majorization would always occur for $|z|<3-\sqrt{8}$ and this was proved by Tao Shah in 1958.

In this paper we show that the result is actually true for functions in $\mathfrak{u}_{\alpha}$ and obtain the sharp radius of majorization as $\alpha+1-\left(\alpha^{2}+2 \alpha\right)^{1 / 2}$ for $1.65 \leq \alpha$ $<\infty$. This yields $3-\sqrt{8}$ for the case $\alpha=2$.

Our investigation shows that the important datum for majorization-subordination theory is not univalence, but the order of a linear invariant family. In particular, many classically derived estimates for univalent functions are true for functions of infinite valence.

Received by the editors September 21, 1973.

AMS (MOS) subject classifications (1970). Primary 30A36, 30A40.

Key words and phrases. Linear invariant family, univalent analytic function, majorization, order of a linear invariant family, subordination. 
The method of proof uses a considerable number of estimates. Because of these estimates it remains an open question as to whether the result of Theorem 1 is true for $1 \leq \alpha<1.65$. We conjecture that Theorem 1 is true in this range, and therefore conjecture that for convex univalent functions $\left(F(z) \in \mathfrak{u}_{1}\right)$ the radius of majorization of the derivative should be $2-\sqrt{3}$.

II. Statement and proof of the theorem. We first state and prove an improved form of the Schwarz lemma for unimodular analytic functions which is due to Tao Shah [5]. We then state a weaker form due to Goluzin.

Lemma 1. Let $\varphi(z)=a z+\cdots, a \geq 0,|\varphi(z)| \leq 1$, be analytic in $|z|<1$. Then

$$
\varphi(z)=z \cdot \frac{a+\omega(z)}{1+a \omega(z)},
$$

where $\omega(z)$ is analytic and satisfies $|\omega(z)| \leq|z|$ in $|z|<1$. Moreover, for any $z_{0}$ in $|z|<1$, if we let $\omega\left(z_{0}\right)=c$, then

$$
\left|\varphi^{\prime}\left(z_{0}\right)\right| \leq\left|\frac{a+2 c+a c^{2}}{(1+a c)^{2}}\right|+\frac{1-a^{2}}{|1+a c|^{2}} \cdot \frac{\left|z_{0}\right|^{2}-|c|^{2}}{1-\left|z_{0}\right|^{2}} .
$$

Proof. Since $|\varphi(z) / z| \leq 1$ in $|z|<1$, the function

$$
\omega(z)=\frac{\varphi(z) / z-a}{1-a \varphi(z) / z}
$$

satisfies the Schwarz lemma. Solving (3) for $\varphi(z)$ yields (1).

Fix a point $z_{0}$ in $D$ and let $\omega\left(z_{0}\right)=c$. The derivative of $\varphi(z)$ at $z_{0}$ is

$$
\varphi^{\prime}\left(z_{0}\right)=\left(z_{0} \omega^{\prime}\left(z_{0}\right)-c\right) \frac{\left(1-a^{2}\right)}{(1+a c)^{2}}+\frac{a+c}{1+a c}+\frac{c\left(1-a^{2}\right)}{(1+a c)^{2}} .
$$

It therefore suffices to show

$$
\left|z_{0} \omega^{\prime}\left(z_{0}\right)-c\right| \leq\left(\left|z_{0}\right|^{2}-|c|^{2}\right) /\left(1-\left|z_{0}\right|^{2}\right) .
$$

The function

$$
f(\zeta)=\left\{\omega\left(\frac{\zeta+z_{0}}{1+\bar{z}_{0} \zeta}\right)-\omega\left(z_{0}\right)\right\} /\left\{1-\overline{\omega\left(z_{0}\right)} \omega\left(\frac{\zeta+z_{0}}{1+\bar{z}_{0} \zeta}\right)\right\}
$$

satisfies the Schwarz lemma in $|\zeta|<1$ and $f\left(-z_{0}\right)=-c$. Let $g(\zeta)=f(\zeta) / \zeta$ and $h(\zeta)=\left(g(\zeta)-f^{\prime}(0)\right) \cdot\left(1-\overline{f^{\prime}(0)} g(\zeta)\right)^{-1}$. Since $h(\zeta)$ also satisfies the Schwarz lemma we obtain

$$
\left|h\left(-z_{0}\right)\right|=\left|\frac{c-z_{0} f^{\prime}(0)}{z_{0}-c f^{\prime}(0)}\right| \leq\left|z_{0}\right| .
$$

However, $f^{\prime}(0)=\left(1-\left|z_{0}\right|^{2}\right)\left(1-|c|^{2}\right)^{-1} \omega^{\prime}\left(z_{0}\right)$ and therefore upon squaring both sides of (5) and noting that 


$$
\left|z_{0}\right|^{2}\left|\omega^{\prime}\left(z_{0}\right)\right|^{2}+|c|^{2}-\left|z_{0} \omega^{\prime}\left(z_{0}\right)-c\right|^{2}=\overline{\omega^{\prime}\left(z_{0}\right)} \bar{z}_{0} c+\bar{c} z_{0} \omega^{\prime}\left(z_{0}\right),
$$

we obtain

$$
\left(1-\left|z_{0}\right|^{2}\right)^{2}\left(\left|z_{0} \omega^{\prime}\left(z_{0}\right)-c\right|^{2}-|c|^{2}\right) \leq\left(1-|c|^{2}\right)\left(\left|z_{0}\right|^{4}-|c|^{2}\right) .
$$

Hence

$$
\left(1-\left|z_{0}\right|^{2}\right)^{2}\left(\left|z_{0} \omega^{\prime}\left(z_{0}\right)-c\right|^{2}\right) \leq\left(\left|z_{0}\right|^{2}-|c|^{2}\right)^{2},
$$

or, equivalently,

$$
\left|z_{0} \omega^{\prime}\left(z_{0}\right)-c\right| \leq\left(\left|z_{0}\right|^{2}-|c|^{2}\right) /\left(1-\left|z_{0}\right|^{2}\right),
$$

which toncludes the lemma.

Lemma 2. Under the condition of Lemma 1,

$$
\left|\frac{\varphi(z)-z}{1-\bar{z} \varphi(z)}\right| \leq \frac{|z|(1-a)}{1+|z|^{2}-|z|(1+a)}, \quad z \in D,
$$

and

$$
\left|\varphi^{\prime}(z)\right| \leq \frac{a\left(1+|z|^{2}\right)+2|z|}{1+|z|^{2}+2 a|z|} \cdot \frac{1-|\varphi(z)|^{2}}{1-|z|^{2}}, \quad z \in D .
$$

Proof. The proof of this lemma can be found within a proof by Goluzin [3,pp. 331-332].

Theorem. Let $f(z)$ be subordinate to $F(z)$ in $D$ with $f^{\prime}(0) \geq 0$. If $F(z) \in \mathfrak{U}_{\alpha}$, $1.65 \leq \alpha<\infty$, then $f^{\prime}(z)$ is majorized by $F^{\prime}(z)$ in $|z| \leq \alpha+1-\left(\alpha^{2}+2 \alpha\right)^{1 / 2}$ and the result is best possible.

Proof. Since $f(z)$ is subordinate to $F(z)$ in $D$ with $f^{\prime}(0) \geq 0$ we have $f(z)=F(\varphi(z))$ where $\varphi(z)$ satisfies Lemma 1 . Choose and fix an arbitrary $z_{0}$ in $|z| \leq(\alpha+1)-\left(\alpha^{2}+2 \alpha\right)^{1 / 2}$. Our goal is to show that $\left|f^{\prime}\left(z_{0}\right) / F^{\prime}\left(z_{0}\right)\right| \leq 1$.

Since $f(z)=F(\varphi(z))$ we have

$$
\left|f^{\prime}\left(z_{0}\right) / F^{\prime}\left(z_{0}\right)\right|=\left|F^{\prime}\left(\varphi\left(z_{0}\right)\right) / F^{\prime}\left(z_{0}\right)\right|\left|\varphi^{\prime}\left(z_{0}\right)\right| .
$$

For any $a$ and $b$ in $D$ and any function $F$ in $\mathfrak{u}_{\alpha}$ we have [4, Lemma 2.1]

$$
\left|\frac{F^{\prime}(a)}{F^{\prime}(b)}\right| \leq \frac{1-|b|^{2}}{1-|a|^{2}}\left(\frac{|1-\bar{a} b|+|a-b|}{|1-\bar{a} b|-|a-b|}\right)^{\alpha} .
$$

We therefore obtain our fundamental inequality

$$
\left|\frac{f^{\prime}\left(z_{0}\right)}{F^{\prime}\left(z_{0}\right)}\right| \leq \frac{1-\left|z_{0}\right|^{2}}{1-\left|\varphi\left(z_{0}\right)\right|^{2}}\left(\frac{\left|1-\overline{\varphi\left(z_{0}\right)} z_{0}\right|+\left|\varphi\left(z_{0}\right)-z_{0}\right|}{\left|1-\overline{\varphi\left(z_{0}\right)} z_{0}\right|-\left|\varphi\left(z_{0}\right)-z_{0}\right|}\right)^{\alpha}\left|\varphi^{\prime}\left(z_{0}\right)\right| .
$$


Our proof now proceeds in two different directions depending on whether $f^{\prime}(0)$ is large or small in relation to $\alpha$. We first consider the case of small $f^{\prime}(0)$; namely,

$$
\begin{aligned}
& 0 \leq f^{\prime}(0) \leq 3 / 20 \quad(\text { if } 1.65 \leq \alpha \leq 2), \\
& \left.0 \leq f^{\prime}(0) \leq 1 / 6 \quad \text { (if } 2 \leq \alpha \leq 3\right), \\
& 0 \leq f^{\prime}(0) \leq 1 / 10 \quad(\text { if } 3 \leq \alpha<\infty) .
\end{aligned}
$$

If we apply Lemma 2 to our fundamental inequality (8) we obtain

$$
\left|\frac{f^{\prime}\left(z_{0}\right)}{F^{\prime}\left(z_{0}\right)}\right| \leq \frac{b a+1}{b+a}\left(\frac{b-a}{b-1}\right)^{\alpha} \equiv k(a, \alpha, b)
$$

where $b=\left(1+\left|z_{0}\right|^{2}\right) / 2\left|z_{0}\right|$ and $a=f^{\prime}(0)$. Note that $b$ is always bounded below by $\alpha+1$ since $r_{0}=\left|z_{0}\right|$ is bounded above by $\alpha+1-\left(\alpha^{2}+2 \alpha\right)^{1 / 2}$.

It is quite easy to show that $k(a, \alpha, b)$ is the product of two positive decreasing functions in $\mathbf{b}$ and hence is itself a decreasing function in $\mathbf{b}$. We now show that $k(a, \alpha, \alpha+1)$ is increasing in a. Since

$$
\frac{\partial k(a, \alpha, \alpha+1)}{\partial a}=\frac{(\alpha+1-a)^{\alpha-1}}{(\alpha)^{\alpha}} \frac{P(a, \alpha)}{(\alpha+1+a)^{2}},
$$

where $P(a, \alpha)=-\alpha(\alpha+1) a^{2}-\left(\alpha^{3}+3 \alpha^{2}+4 \alpha\right) a+\alpha(\alpha+1)^{2}$, we are reduced to establishing $P(a, \alpha) \geq 0$. But $P(a, \alpha)$ is a quadratic in a with negative leading coefficient and $P(0, \alpha)>0$. Therefore, if $P(.4, \alpha)$ is greater than $0, P(a, \alpha)$ will be greater than zero for any $a$ in $[0, .4]$ which will conclude the argument that $P(a, \alpha)$ is nonnegative for small $f^{\prime}(0)$.

A computation shows $P(.4, \alpha+1)=\alpha\left(.6 \alpha^{2}+.64 \alpha-.76\right)>0$ which therefore concludes the demonstration that $k(a, \alpha, \alpha+1)$ is increasing in a. Thus $\left|f^{\prime}\left(z_{0}\right) / F^{\prime}\left(z_{0}\right)\right| \leq k(a, \alpha, b) \leq k(a, \alpha, \alpha+1)$, and, since $k(a, \alpha, \alpha+1)$ is increasing in a, in order to conclude the proof of the theorem for 'small' values of $a$, it suffices to show that

(a) $k(3 / 20, \alpha, \alpha+1) \leq 1$ when $1.65 \leq \alpha \leq 2$,

(b) $k(1 / 6, \alpha, \alpha+1) \leq 1$ when $2 \leq \alpha \leq 3$,

(c) $k(1 / 10, \alpha, \alpha+1) \leq 1$ when $3 \leq \alpha<\infty$.

Subcase a. Since

$(d / d \alpha) k(.15, \alpha, \alpha+1)$

$$
\begin{aligned}
& =\left(\frac{\alpha+1-.15}{\alpha}\right)^{\alpha}\left[\frac{(.15)^{2}-1}{(\alpha+.15+1)^{2}}\right. \\
& \left.+\frac{(\alpha+1) .15+1}{\alpha+.15+1}\left(\log \frac{\alpha+1-.15}{\alpha}+\frac{.15-1}{\alpha+1-.15}\right)\right] \\
& \leq\left(\frac{\alpha+1-.15}{\alpha}\right)^{\alpha}\left[\frac{(.15)^{2}-1}{(2+.15+1)^{2}}+\frac{(1.65+1) .15+1}{1.65+.15+1}\left(\log \frac{1.65+1-.15}{1.65}\right)\right. \\
& \left.+\left(\frac{(2+1) .15+1}{2+.15+1}\right)\left(\frac{.15-1}{2+1-.15}\right)\right]
\end{aligned}
$$


for $1.65 \leq \alpha \leq 2$, therefore $k(.15, \alpha, \alpha+1)$ is a decreasing function of $\alpha$ in this range. It therefore suffices to check by a routine computation that $k(.15,1.65$, $2.65) \leq 1$.

Subcase b. Since $[(\alpha+1) a+1] /(\alpha+a+1)$ is decreasing with $\alpha$, and $((\alpha$ $+b) / \alpha)^{\alpha}$ is an increasing function of $\alpha$ for all $\alpha>0$ and all $b>0$, we can state

$$
k(1 / 6, \alpha, \alpha+1) \leq \frac{(2+1)(1 / 6)+1}{2+1 / 6+1}\left(\frac{3+1-1 / 6}{3}\right)^{3} .
$$

It is easy to verify that this latter quantity is indeed less than one.

Subcase c. This is the easiest case since, as above,

$$
k(.10, \alpha, \alpha+1) \leq e\left(\frac{(3+1) \cdot 10+1}{3+.10+1}\right)<1 .
$$

Thus for small values of $f^{\prime}(0)$ we have shown that $f^{\prime}(z)$ is majorized by $F^{\prime}(z)$ in $|z|<(\alpha+1)-\left(\alpha^{2}+2 \alpha\right)^{1 / 2}$.

We now consider the case that $f^{\prime}(0)$ is large. Returning to our fundamental inequality (8), we note that in the language of Lemma 1

$$
\begin{aligned}
& \quad \varphi\left(z_{0}\right)=z_{0}(a+c) /(1+a c), \quad c=r e^{i \theta},\left|z_{0}\right|=r_{0}, \text { and } \\
& \left(1-\left|z_{0}\right|^{2}\right)\left(1-\left|\varphi\left(z_{0}\right)\right|^{2}\right) \\
& =|1+a c|^{-2}\left(\left|1+a c-r_{0}^{2}(a+c)\right|+r_{0}(1-a)|1-c|\right) \\
& \cdot\left(\left|1+a c-r_{0}^{2}(a+c)\right|-r_{0}(1-a)|1-c|\right) .
\end{aligned}
$$

Therefore (8), (10) and Lemma 1 together imply that

$$
\begin{aligned}
\left|\frac{f^{\prime}\left(z_{0}\right)}{F^{\prime}\left(z_{0}\right)}\right| \leq & \left(1-r_{0}^{2}\right) \frac{\left(\left|1+a c-r_{0}^{2}(a+c)\right|+r_{0}(1-a)|1-c|\right)^{\alpha-1}}{\left(\left|1+a c-r_{0}^{2}(a+c)\right|-r_{0}(1+a)|1-c|\right)^{\alpha+1}} \\
& \cdot\left[\left|a+2 c+a c^{2}\right|\left(1-r_{0}^{2}\right)+\left(r_{0}^{2}-|c|^{2}\right)\left(1-a^{2}\right)\right] .
\end{aligned}
$$

Lemma 3, at the end of the paper, shows that when $f^{\prime}(0)$ is 'large' the right-hand side of (11) as a function of $c=r e^{i \theta}$ has its maximum at $\theta=0$. Noting that

$$
1+a r-r_{0}^{2}(a+r) \pm r_{0}(1-a)(1-r)=\left(1 \pm r_{0}\right)\left[1+a r \mp r_{0}(a+r)\right],
$$

we infer

$$
\begin{aligned}
\left|\frac{f^{\prime}\left(z_{0}\right)}{F^{\prime}\left(z_{0}\right)}\right| \leq & \left(\frac{1+r_{0}}{1-r_{0}}\right)^{\alpha} \frac{\left[1+a r-r_{0}(a+r)\right]^{\alpha-1}}{\left[1+a r+r_{0}(a+r)\right]^{\alpha+1}} \\
& \cdot\left[\left(1-r_{0}^{2}\right)\left(a+2 r+a r^{2}\right)+\left(r_{0}^{2}-r^{2}\right)\left(1-a^{2}\right)\right] .
\end{aligned}
$$

Let $L\left(r, r_{0}, a\right)$ denote the right-hand side of (12). The proof of majorization will be concluded if we can show that $L$ is an increasing function of a since $L\left(r, r_{0}, 1\right) \equiv 1$. 
However,

$$
\frac{d L}{d a}=\left(\frac{1+r_{0}}{1-r_{0}}\right)^{\alpha} \frac{\left[1+a r-r_{0}(a+r)\right]^{\alpha-2}}{\left[1+a r+r_{0}(a+r)\right]^{\alpha+2}} \cdot R(a)
$$

where

$$
\begin{aligned}
R(a)= & {\left[(1+a r)^{2}-r_{0}(a+r)^{2}\right] \cdot\left[1-r_{0}^{2} r^{2}-(2 a+1)\left(r_{0}^{2}-r^{2}\right)\right] } \\
& -\left[\left(1-r_{0}^{2}\right)\left(a+2 r+a r^{2}\right)+\left(1-a^{2}\right)\left(r_{0}^{2}-r^{2}\right)\right] \\
& \cdot\left[2 r\left(1-r_{0}^{2}\right)+2 \alpha r_{0}\left(1-r^{2}\right)-2 a\left(r_{0}^{2}-r^{2}\right)\right] .
\end{aligned}
$$

The problem then is to show $R(a)$ is nonnegative. Since

$$
\begin{aligned}
R^{\prime}(a)= & 2\left(r_{0}^{2}-r^{2}\right)\left[2 \alpha a r_{0}\left(1-r^{2}\right)+(1-r)^{2}\left(1-r_{0}^{2}\right)(a-1)\right] \\
& -2 \alpha r_{0} \cdot\left(1-r_{0}^{2}\right)\left(1-r^{4}\right) \\
\leq & 2 \alpha r_{0}\left[2 r_{0}^{2}-\left(1-r_{0}^{2}\right)\left(1-r_{0}^{4}\right)\right],
\end{aligned}
$$

we can conclude that $R(a)$ is a decreasing function if we note that $2 r_{0}^{2}$ $-\left(1-r_{0}^{2}\right)\left(1-r_{0}^{4}\right)<0$ since $r_{0} \leq(\alpha+1)-\left(\alpha^{2}+2 \alpha\right)^{1 / 2} \leq 1 / 2$. Thus $R(a)$ $\geq R(1)$. However,

$$
\begin{aligned}
R(1) & =(1+r)^{2}\left(1-r_{0}^{2}\right)(1-r)\left[(1-r)\left(1-r_{0}^{2}\right)-2 \alpha r_{0}(1+r)\right] \\
& \geq(1+r)^{2}\left(1-r_{0}^{2}\right)(1-r)\left(1+r_{0}\right)\left[1-r_{0}^{2}-2 \alpha r_{0}\right] \\
& \geq 0,
\end{aligned}
$$

since $\left(1-r_{0}^{2}\right)-2 \alpha r_{0} \geq 0$ for $r_{0} \leq(\alpha+1)-\left(\alpha^{2}+2 \alpha\right)^{1 / 2}$.

Thus for large $f^{\prime}(0),\left|f^{\prime}\left(z_{0}\right) / F^{\prime}\left(z_{0}\right)\right| \leq 1$; that is, $f^{\prime}(z)$ is majorized by $F^{\prime}(z)$ in $|z| \leq(\alpha+1)-\left(\alpha^{2}+2 \alpha\right)^{1 / 2}$.

We now show that this result cannot be improved. This means that for any real number $m^{\prime}>m(\alpha) \equiv(\alpha+1)-\left(\alpha^{2}+2 \alpha\right)^{1 / 2}$ we must find analytic functions $f(z)$ and $F(z)$ such that $f(z)$ is subordinate to $F(z), f^{\prime}(0) \geq 0, F(z) \in \mathfrak{U}_{\alpha}$, but for which $\left|f^{\prime}(z)\right| \leq\left|F^{\prime}(z)\right|$ for all $|z|<m^{\prime}$ is false.

Let

$$
F(z)=\frac{1}{2 \alpha}\left\{1-\left(\frac{1-z}{1+z}\right)^{\alpha}\right\} \text { and } f(z, a)=F(\varphi(z))
$$

where $\varphi(z)=z(a+z) /(1+a z), 0 \leq a \leq 1$. Then $f(z, a)$ is subordinate to $F(z)$ in $D$ for any a, $0 \leq a \leq 1, F(z)$ is in $\mathfrak{U}_{\alpha}$, and $f^{\prime}(0) \geq 0$. A computation shows

$$
\left.\frac{\partial}{\partial z} f(z, a)\right|_{z=r}={\frac{\left(1-r^{2}\right)^{\alpha-1}}{2^{\alpha} r^{\alpha}}} \cdot \frac{a b+1}{(b+a)^{\alpha+1}}
$$

where $b=\left(1+r^{2}\right) / 2 r$, and 


$$
\left.\frac{\partial}{\partial a}\left[\left.\frac{\partial}{\partial z} f(z, a)\right|_{z=r}\right]\right|_{a=1}=\frac{\left(1-r^{2}\right)^{\alpha-1}}{2^{\alpha} r^{\alpha}} \cdot \frac{[b-(\alpha+1)]}{(b+1)^{\alpha+1}} .
$$

Thus if we let $z=r, m<r<m^{\prime}$, then $b=\left(1+r^{2}\right) / 2 r<\alpha+1$ and (13) implies that $\partial f(z, a) /\left.\partial z\right|_{z=r}$ is a decreasing function of a for such a value of $r$. Therefore for a sufficiently close to 1 ,

$$
f^{\prime}(r, a) \equiv \partial f(z, a) /\left.\partial z\right|_{z=r}>\partial f(z, 1) /\left.\partial z\right|_{z=r} \equiv F^{\prime}(r)>0 .
$$

Therefore $f^{\prime}$ is not majorized by $F^{\prime}$ in $|z|<m^{\prime}$.

This concludes the proof of the theorem.

Corollary 1. If $f(z)$ is majorized by $F(z)$ in $\mathfrak{u}_{2}$ and $f^{\prime}(0) \geq 0$, then $f^{\prime}(z)$ is majorized by $F^{\prime}(z)$ in $|z| \leq 3-\sqrt{8}$ and the result is sharp.

Corollary 1 is an improvement on Tao Shah's result for $F(z)$ in $\subseteq$ since $\subseteq$ is a proper subset of $\mathfrak{U}_{2}$. The same estimates therefore hold even for the functions of infinite valence which lie in $\mathfrak{U}_{2}$.

\section{Statement and proof of Lemma 3.}

Lemma 3. If $1.65 \leq \alpha \leq 2$ and $3 / 20 \leq a \leq 1$, or if $2 \leq \alpha \leq 3$ and $1 / 6 \leq a$ $\leq 1$, or if $3 \leq \alpha \leq \infty$ and $1 / 10 \leq a \leq 1$, then, as a function of $\theta$, the maximum of

$$
\begin{aligned}
\frac{\left(\left|1+a c-r_{0}^{2}(a+c)\right|+r_{0}(1-a)|1-c|\right)^{\alpha-1}}{\left(\left|1+a c-r_{0}^{2}(a+c)\right|-r_{0}(1-a)|1-c|\right)^{\alpha+1}} & \\
\cdot & \left\{\left|a+2 c+a c^{2}\right|\left(1-r_{0}^{2}\right)+\left(r_{0}^{2}-|c|^{2}\right)\left(1-a^{2}\right)\right\},
\end{aligned}
$$

where $c=r e^{i \theta}$ and $0 \leq r \leq r_{0} \leq \alpha+1-\left(\alpha^{2}+2 \alpha\right)^{1 / 2}$, occurs at $\theta=0$.

Proof. Let $I(\theta)$ denote the quantity in (14). In order to compute $d I / d \theta$ we first write $I$ as $I=A^{\alpha-1} C / B^{\alpha+1}$ where

$$
\begin{aligned}
& A \equiv\left|1-a r_{0}^{2}+c\left(a-r_{0}^{2}\right)\right|+r_{0}(1-a)|1-c| \equiv D+E, \\
& B \equiv\left|1-a r_{0}^{2}+c\left(a-r_{0}^{2}\right)\right|-r_{0}(1-a)|1-c| \equiv D-E, \\
& C \equiv\left|a+2 c+a c^{2}\right|\left(1-r_{0}^{2}\right)+\left(r_{0}^{2}-r^{2}\right)\left(1-a^{2}\right) .
\end{aligned}
$$

Then

$$
\frac{d I}{d \theta}=\frac{A^{\alpha-2}}{B^{\alpha+2}}\left[\left\{A B \frac{d C}{d \theta}+2 C\left(\frac{E d E}{d \theta}-\frac{D d D}{d \theta}\right)\right\}+2 \alpha C\left(\frac{D d E}{d \theta}-\frac{E d D}{d \theta}\right)\right]
$$

Since

$$
\begin{aligned}
& \frac{d D}{d \theta}=\frac{-r \sin \theta}{D}\left(1-a r_{0}^{2}\right)\left(a-r_{0}^{2}\right), \\
& \frac{d E}{d \theta}=\frac{r \sin \theta}{E}
\end{aligned}
$$




$$
\begin{aligned}
& \frac{d C}{d \theta}=\frac{-2 a r \sin \theta}{\left|a+2 c+a c^{2}\right|}\left(1-r_{0}^{2}\right)\left(1+r^{2}+2 a r \cos \theta\right), \\
& A B=\left(1-r_{0}^{2}\right)\left[1-a^{2} r_{0}^{2}+r^{2}\left(a^{2}-r_{0}^{2}\right)+\left(1-r_{0}^{2}\right) 2 a r \cos \theta\right], \\
& E \frac{d E}{d \theta}-D \frac{d D}{d \theta}=a\left(1-r_{0}^{2}\right)^{2} r \sin \theta, \\
& D \frac{d E}{d \theta}-E \frac{d D}{d \theta}=\frac{r r_{0} \sin \theta\left(1-a^{2}\right)}{D E}\left(1-r_{0}^{2}\right)\left(1-a r_{0}^{2}+r^{2}\left(a-r_{0}^{2}\right)\right),
\end{aligned}
$$

we can verify that

$$
\frac{d I}{d \theta}=\frac{A^{\alpha-2}}{B^{\alpha+2}}\left(\frac{-2 a r \sin \theta\left(1-r_{0}^{2}\right)^{2}\left(1-a^{2}\right)}{\left|a+2 c+a c^{2}\right|}\right)\left\{I_{1}+I_{2}+I_{3}\right\},
$$

where

$$
\begin{aligned}
I_{1}= & \left(1-r^{2}\right)\left(1+r^{2} r_{0}^{2}\right)-2 r^{2}\left(1-r_{0}^{2}\right)+2 a r\left(r_{0}^{2}-r^{2}\right) \cos \theta, \\
I_{2}= & -\left(r_{0}^{2}-r^{2}\right)\left|a+2 c+a c^{2}\right|, \\
I_{3}= & \frac{-\alpha r_{0}}{a\left(1-r_{0}^{2}\right)}\left\{\frac{1-a r_{0}^{2}+r^{2}\left(a-r_{0}^{2}\right)}{|1-c|\left|1-a r_{0}^{2}+c\left(a-r_{0}^{2}\right)\right|}\right\} \\
& \cdot\left\{\left(1-a^{2}\right)\left(r_{0}^{2}-r^{2}\right)\left|a+2 c+a c^{2}\right|+\left|a+2 c+a c^{2}\right|^{2}\left(1-r_{0}^{2}\right)\right\} .
\end{aligned}
$$

Clearly it now suffices to verify that $I_{1}+I_{2}+I_{3}>0$ in order to prove the maximum of $I(\theta)$ occurs at $\theta=0$.

We first determine an estimate for $I_{3}$. The expression in the denominator of $I_{3}$ satisfies

$$
|1-c|\left|1-a r_{0}^{2}+c\left(a-r_{0}^{2}\right)\right| \geq(1-r)\left(1-a r_{0}^{2}+r\left(a-r_{0}^{2}\right)\right) .
$$

This is most easily seen by squaring both expressions, removing the common factors and noting that $0<\left(a-r_{0}^{2}\right) /\left(1-a r_{0}^{2}\right) \leq 1$ since $a \geq .10>(\alpha+1$ $\left.-\left(\alpha^{2}+2 \alpha\right)^{1 / 2}\right)^{2} \geq r_{0}^{2}$. Thus,

$$
\begin{aligned}
\left|I_{3}\right| \leq & \frac{\alpha r_{0}\left[1-a r_{0}^{2}+r^{2}\left(a-r_{0}^{2}\right)\right]\left(a+2 r+a r^{2}\right)}{a\left(1-r_{0}^{2}\right)(1-r)\left(1-a r_{0}^{2}+r\left(a-r_{0}^{2}\right)\right)} \\
& \cdot\left\{\left(1-a^{2}\right)\left(r_{0}^{2}-r^{2}\right)+\left(a+2 r+a r^{2}\right)\left(1-r_{0}^{2}\right)\right\} .
\end{aligned}
$$

The denominator of (16) is a decreasing function of $r$ and the numerator of (16) is the product of three increasing functions in $r$. Consequently we obtain,

$$
\left|I_{3}\right| \leq \frac{\alpha r_{0}}{a} \frac{\left(1+r_{0}^{2}\right)\left(1+r_{0}\right)}{\left(1-r_{0}\right)} \frac{\left[a\left(1+r_{0}^{2}\right)+2 r_{0}\right]^{2}}{\left(1+r_{0}^{2}+r_{0}(1+a)\right)}=J_{3} .
$$

However, 


$$
\frac{d}{d r_{0}}\left(\frac{a\left(1+r_{0}^{2}\right)+2 r_{0}}{1+r_{0}^{2}+r_{0}(1+a)}\right)=\frac{\left(1-r_{0}^{2}\right)[2-a(1+a)]}{\left(1+r_{0}^{2}+r_{0}(1+a)\right)^{2}} \geq 0 .
$$

Therefore $J_{3}$ is the product of monotone increasing functions in $r_{0}$ and so $J_{3} \leq\left. J_{3}\right|_{r_{0}=m}$, where $m=m(\alpha)=\alpha+1-\left(\alpha^{2}+2 \alpha\right)^{1 / 2}$. Upon substituting this value for $r_{0}$ into $J_{3}$ and noting that $1+m^{2}=2(\alpha+1) m$, we obtain

$$
\left|I_{3}\right| \leq \frac{8(\alpha+1)}{a} \alpha m^{3} \frac{(1+m)}{1-m} \frac{[1+a(\alpha+1)]^{2}}{(3+a+2 \alpha)}=J_{4} .
$$

We now turn to a lower estimate on $I_{1}+I_{2}$. Clearly,

$$
I_{1}+I_{2} \geq J_{5} \equiv\left(1-r^{2}\right)\left(1+r_{0}^{2} r^{2}\right)-2 r^{2}\left(1-r_{0}^{2}\right)-\left(r_{0}^{2}-r^{2}\right)\left(1+4 r+r^{2}\right) .
$$

To obtain a lower estimate for $J_{5}$ we note that

$$
\begin{aligned}
d J_{5} / d r & =-4\left[r_{0}^{2}+r\left(1-r^{2}-r_{0}^{2}+r^{2} r_{0}^{2}-3 r\right)\right] \\
& <-4\left[r\left(1-2 r_{0}^{2}-3 r_{0}\right)+r_{0}^{2}\right] .
\end{aligned}
$$

Since $1-2 r_{0}^{2}-3 r_{0} \geq 1-2 m^{2}-3 m>.03$, it follows that $J_{5}$ is a decreasing function of $r$. Therefore upon noting $(1-m)^{2}=2 \alpha m$ and $\left(1-m^{2}\right)^{2}=4 m^{2}\left(\alpha^{2}\right.$ $+2 \alpha)$ we can conclude that

$$
J_{5}(r) \geq J_{5}\left(r_{0}\right)=\left(1-r_{0}^{2}\right)^{3} \geq\left(1-m^{2}\right)^{3}=8 m^{3}\left(\alpha^{3}+2 \alpha^{2}\right) \frac{1+m}{1-m} .
$$

Therefore,

$$
I_{1}+I_{2}+I_{3} \geq J_{5}-J_{4} \geq \frac{8 \alpha m^{3}(1+m)}{(1-m) a(3+a+2 \alpha)} \cdot Q(a, \alpha),
$$

where $Q(a, \alpha)=a^{2}\left(-\alpha^{3}-2 \alpha^{2}-\alpha-1\right)+a\left(2 \alpha^{3}+5 \alpha^{2}+2 \alpha-2\right)-(\alpha+1)$. We are therefore reduced to showing $Q(a, \alpha)$ is positive for all possible cases of $a$ and $\alpha$ in the hypothesis of the lemma.

We first note that $Q(a, \alpha)$ is an increasing function of $\alpha$ since

$$
\begin{aligned}
\frac{\partial Q}{\partial \alpha} & =\alpha^{2}\left(6 a-3 a^{2}\right)+\alpha\left(10 a-4 a^{2}\right)-1+2 a-a^{2} \\
& >.57 \alpha^{2}+.96 \alpha-.81>0 .
\end{aligned}
$$

We next note that $Q$ is a quadratic function in a with negative leading coefficient and $Q(1, \alpha)=\alpha^{3}+3 \alpha^{2}-4 \geq 0$. Thus if $Q\left(a_{0}, \alpha\right)>0$ for $a_{0}$ in $(0,1)$, then $Q(a, \alpha)>0$ for all $a, a_{0} \leq a \leq 1$. We thus need only show that $Q(3 / 20,1.65)$, $Q(1 / 6,2)$ and $Q(1 / 10,3)$ are each positive. This is true as a routine computation indicates.

This concludes the proof of the lemma. 


\section{BIBLIOGRAPHY}

1. D. M. Campbell, Majorization-subordination theorems for locally univalent functions, Bull. Amer. Math. Soc. 78 (1972), 535-538. MR 45 \#8817.

2. , Majorization-subordination theorems for locally univalent functions. II, Canad. J. Math. 25 (1973), 420-425.

3. G. M. Goluzin, Geometric theory of functions of a complex variable, GITTL, Moscow, 1952; English transl., Transl. Math. Monographs, vol. 26, Amer. Math. Soc., Providence, R. I., 1969. MR 15, 112; 40 \#308.

4. Ch. Pommerenke, Linear-invariante Familien analytischer Funktionen. I, Math. Ann. 155 (1964), 108-154. MR 29 \#6007.

5. Tao Shah, On the radius of superiority in subordination, Sci. Record 1 (1957), 329-333. MR 20 \#6531.

Department of Mathematics, Brigham Young Universtty, Provo, Utah 84602 